\section{Analytik von polyzyklischen aromatischen Kohlenwasserstoffen (PAK) in imprägnierten Hölzern durch synchrone Fluoreszenzspektroskopie}

\section{T. Salthammer}

Wilhelm-Klauditz-Institut (WKI), Fraunhofer-Arbeitsgruppe für Holzforschung, Bienroder Weg 54E, 3300 Braunschweig

Subject: Analysis of polycyclic hydrocarbons in impregnated wood by synchroneous fluorescence spectroscopy

Material und Methode: Es wurden imprägnicrte Hölzer (i. a. Eisenbahnschwellen) verschiedener Herkunft fluoreszenzspek troskopisch auf polyzyklische aromatische Kohlenwasserstoffe untersucht.

Das Probenmaterial wurde zerkleinert und ca. $6 \mathrm{~g}$ mit $200 \mathrm{ml}$ $\mathrm{n}$-Hexan in einer Soxhletapparatur $7 \mathrm{~h}$ extrahiert. Das Lösungsmittel wurde im Vakuumrotationsverdampfer abgezogen und der Extraktgehalt ermittelt. Der Rückstand wurde in $\mathrm{n}$-Hexan neu aufgenommen und bis zu einer Extinktion $<0.5$ in Absorptionsmaximum verdünnt. Fluoreszenzspektren wurden mit dem sogenannten Synchronverfahren aufgenommen. Bei dieser Methode werden Anregungs- und Enissionswellenlänge im Gegensatz zur herkömmlichen statischen Fluoreszenzspektroskopie kontinuierlich mit einem konstanten Abstand. $\Delta \lambda$. durchlaufen ${ }^{1}$ ).

Ergebnisse: Die Abbildung zeigt das mit $\Delta \lambda=10 \mathrm{~nm}$ synchron detektierte Fluoreszenzspektrum eines Holzextraktes. Die im Wellenlängenbereich von $300-500 \mathrm{~nm}$ gut fluoreszierenden Aromaten (hier: Phenanthren, Pyren, Anthracen und Perylen) werden mit hoher Empfindlichkeit und Selektivität nachgewiesen. Diese Verbindungen sind in Carbolineen stark repräsenticrt und können somit als Leitsubstanzen dienen. Das Verfahren zeichnet sich vor allem durch eine kurze Analysenzeit aus und erlaubt eine schnelle und sichere qualitative Beurteilung von imprägnierten Hölzern.

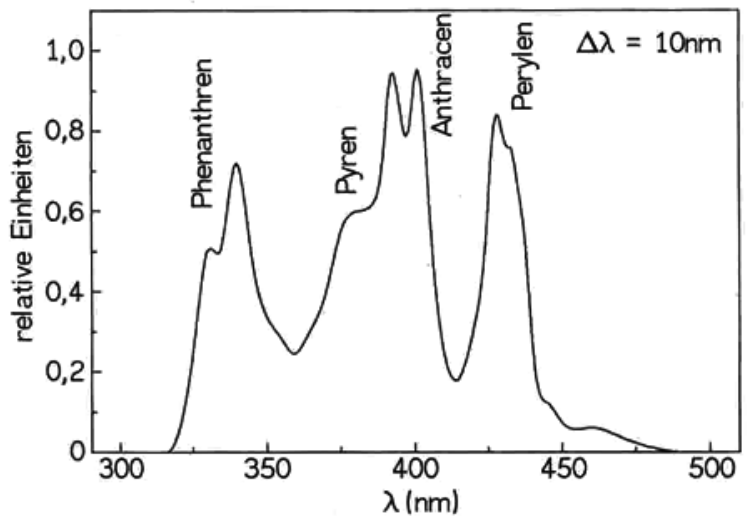

1) T. Vo-Dinh, Anal. Chem. 50 (1978) 396

\section{Acetat- und Formiatgehalte im Splintholzsaft der Fichte}

\section{E. Roffael; H. Miertzsch: T. Schwarz}

Fraunhofer-Arbeitsgruppe für Holzforschung (WKI) Bienroder Weg 54 E, W-3300 Braunschweig

Subject: The content of acetate and formiate ions in the sap of spruce sapwood depends on felling time, tree age, and height of test sample.

Material und Methode: Fichrenbäume unterschiedlichen Alters wurden aus demselben Standort (Lonau/Harz) zu unterschiedlichen Jahreszeiten (Februar, A pril, Juli) gefällt. Unmittelbar danach wurde Saft aus dem Splintholz in unterschiedlicher Baumhöhe (vgl. WKI$\mathrm{Kb}-\mathrm{Nr} .40 / 91$ ) herausgepreßt, für den auch der Gchalt an Formiatund Acetationen ionenchromatographisch bestimmt wurde.

Ergebnisse (s. Tabelle): 1. Der Gehalt an Formiat- und Acelationen nimmt im allgemeinen von Februar über April zum Juli hin deutlich ab. 2. Im Februar verringert sich der Gehalt an Acetat- und Formiationen mit zunehmender Baumhöhe, während im April und Juli keine charakteristischen Unterschiede im Gehalt des Saftes an Formiat- und Acetationen in Abhängigkeit von der Baumhöhe zu erkennen sind. 3. Sowohl der Gehalt an Formiat-als auch an Acetationen lag im Splintholz des alten Baums (136 Jahre) im Jahresdurchschnitt niedriger als bei den 37- und 84jährigen Bäumen.

Veränderungen des Gehaltes an Acetat- und Formiationen (in ppm) in Splintholzsalt der Fichte in Ablängigkeit von Einschlagzeit, Baumalter und Höhe der Probennalime.

\begin{tabular}{|c|c|c|c|c|c|}
\hline \multirow{2}{*}{$\begin{array}{l}\text { Baum- } \\
\text { alter } \\
\text { (Jahre) }\end{array}$} & \multirow{2}{*}{$\begin{array}{l}\text { Proben- } \\
\text { Höhe } \\
\text { (m) }\end{array}$} & \multicolumn{4}{|c|}{$\begin{array}{l}\text { Gehalt an Acetat }(A) \text { und Formiat }(F) \\
\text { in Abhängigkeit von der Einschlagzeit * }\end{array}$} \\
\hline & & & Februar & April & Juli \\
\hline \multirow[t]{6}{*}{37} & 0,5 & $F$ & 200 & 48 & 25 \\
\hline & & $A$ & 143 & 31 & 23 \\
\hline & 2,0 & $\mathrm{~F}$ & 33 & 45 & 2.3 \\
\hline & & A & 105 & 25 & 11. b. \\
\hline & 4,0 & $\mathbf{F}$ & 37 & 50 & 34 \\
\hline & & $\mathrm{A}$ & 54 & 32 & 26 \\
\hline \multirow[t]{6}{*}{84} & 0,5 & $\mathrm{~F}$ & 440 & 31 & 21 \\
\hline & & A & 180 & 30 & 12 \\
\hline & 8,0 & $\mathrm{~F}$ & n. b. & n. b. & n. $b$. \\
\hline & & $A$ & 61,9 & n. b. & n. b. \\
\hline & 16,0 & $\mathrm{~F}$ & 33 & 25 & 20 \\
\hline & & A & 23 & 29 & 15 \\
\hline \multirow[t]{6}{*}{136} & 0,5 & $\mathrm{~F}$ & 68 & 45 & 11 \\
\hline & & A & 81 & 27 & n. b. \\
\hline & 10,0 & $F$ & 90 & 49 & 18 \\
\hline & & A & 46 & 19 & 15 \\
\hline & 20,0 & $F$ & 26 & 35 & 18 \\
\hline & & A & 34 & 15 & 12 \\
\hline
\end{tabular}

* berechnet als freie Säure in ppm; n. b. = nicht bestimmt 